\title{
COMPUTER SIMULATION OF TRAFFIC FLOW AT A SIGNALIZED INTERSECTION
}

\author{
By Sho Myozin*
}

\section{INTRODUCTION}

Owing to the traffic congestion in large towns, the city redevelopment, the reorganization of street networks by constructing parking spaces and urban freeways, and the traffic regulations have been urgent matters to be successful. For the first two matters we should have views upon the generation of traffic demands in whole city, and for the last a microscopic counterplan should be required, i.e. it includes at-grade intersection controls, improvements of road conditions, and the other sorts of traffic regulations. Some of the general methods of at-grade intersection controls are those by stop signs or traffic signals and several kinds of turning regulations.

When we control traffic flow with traffic signal from standpoint of realizing the smooth flow, one of the most important problems is to optimize the cycle time. A deterministic or probabilistic approach has been made to decide it. When, however, we consider the statistical nature of inputs, condition of car movement, and some other conditions, it will be found that those approaches are not always sufficient.

This paper deals with the simulation technique which is one of the powerful tools in an analysis of complex system such as mentioned above, and tries to find the optimal cycle time of traffic light. Ultimately, the optimization of the whole street networks including many intersections connected to each other should be reached. We, however, for the first trial simulate traffic flow at a 2 lane and a 4 lane at-grade intersections equipped with traffic lights.

\section{MODEL DESCRIPTION}

Fig. 1 shows a 2 lane intersection model and Fig. 2 a 4 lane. Each lane under study is

* Assistant of Transportation Engineering, Kyoto University.

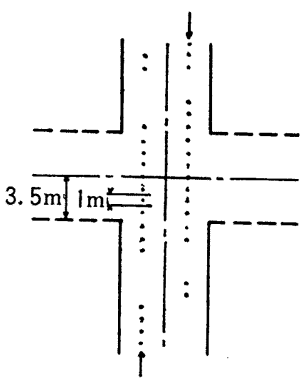

Fig. 1 2-lane intersection model.

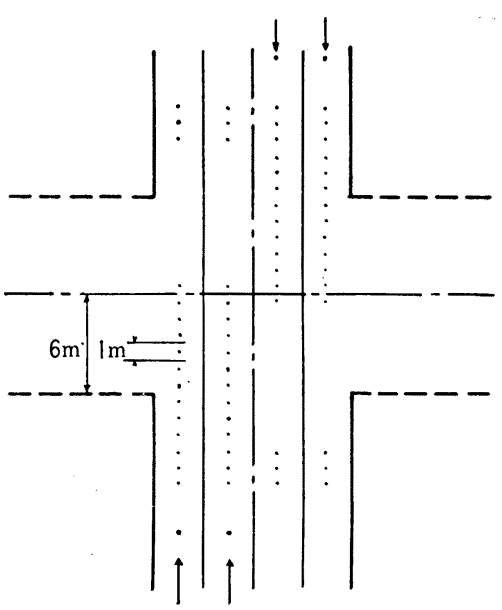

Fig. 2 4-lane intersection model.

01111100011111000000111110000000

Fig. 3 Representation of flow of cars.

divided into small pieces each with equal length 1 meter, which are represented by given co-ordinates. In expressing car movements there are some differences between Fig. 1 and Fig. 2, about which we exp lain in what follows.

By giving figure 1's to several successive coordinates over which one car exists and figure 0 's otherwise, a line of cars at a certain time will be shown, for instance, like Fig. 3 .

In this paper, by supposing every car is uniformly 4 meters in length, we represent a car 
by four successive figure 1's (Fig. 3). Such an expression as Fig. 3 may be called the physical representation, for which it is convenient to use the binary computer. The computer we used is KDC-1 in the Kyoto University Computer Center, which is not binary, but it is possible to use such representation as Fig. 3 by making each computer memory correspond to each co-ordinate.

Upon this, another expression may be called the memorandum representation, which, in short, supplies each car in the system with one memory; i.e. consisting of several cells, the memory can include such items as the existence, driving direction, speed, present co-ordinate of a car in these cells. At every sweeping, we can see any state of a car by decoding one of them memoried in particuler cell or cells.

Now it will be found that motion of each car in the system can be realized by transfering, by the physical representation, contents stored in a certain memory to its front one, or by transforming, by the memorandum one, those in a fixed memory. Needless to say, every moving operation is accompanied by changes of speed or co-ordinates if necessary. Fig. 1, the 2 lane intersection model, is based on the assumption of using the physical representation, and Fig. 2, the 4 lane model, the memorandum one. Each approach is 200 meters long and the front exit of each lane is 50 meters distant from the inlet of the intersection. We have made several assumptions on these intersections. They are;

1. Neglecting the traffic on the orthogonal street. At the intersection equipped with traffic signal it is unnecessary to pay any attention to conflict of traffic flow under study with that on the orthogonal street.

2. Neglecting pedestrians. This may not be actual assumption for the cars turing to right or left, but it seems very difficult to formulate the influence of pedestrians upon them. So, we assume this tentatively.

3. Supposing that the 2 lane intersection has central space or pocket in each direction, which can accomodate two right turning cars at a time, and that 4 lane intersection four cars.
4. Model is not set up of the right and left turning lanes.

Next, we should mention of the model of car movement. The fundamental equation of car moving is as follows,

$$
x_{k}(t-T)-x_{k+1}(t-T)=T \dot{x}_{k+1}(t)+b
$$

where $x_{k}(t)$ and $x_{k+1}(t)$ are respectively the $k$ th and $(k+1)$ st cars' co-ordinates at time $t$ and $\dot{x}_{k+1}(t)$ means $(k+1)$ st car's speed at the sametime. $T$, which is so called the reaction time, is assumed 1 second. We give $b=5$ meters from the fact that every car has been assumed 4 meters in length.

According to this fundamental equation, the $k$ th car from the head of waiting line is to start at $k x T=k$ seconds after the light has changed from red to green. This will be obvious from the fact that the 1 st car's reaction time should be 1 second as well as the others'.

We have modeled every car's acceleration like Fig. 4, i.e., uniformly for every car we have assumed the accelerating speed $2 \mathrm{~m} / \mathrm{sec}^{2}$ and the travelling speed, during time $t$ and $t+1$, of $(2 t+1) \mathrm{m} / \mathrm{sec}$, the average in the interval. Moreover, we assume no car can get speed over $10 \mathrm{~m} / \mathrm{sec}$. Once a car has reached the maximum speed of $10 \mathrm{~m} / \mathrm{sec}$ it accelerates no longer and continues travelling at the speed, following the leading car from about 15 meters behind. It will be released out of the system at last when it has reached the exit co-ordinate.

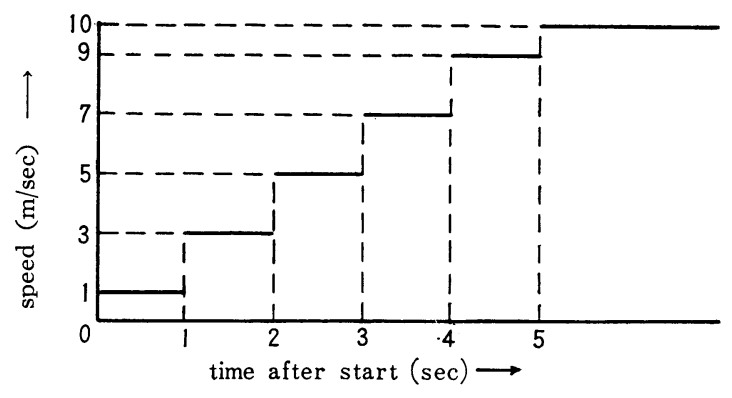

Fig. 4 Acceleration model.

Now, let's see how a car generated joins to the queue. The cars which are generated during red hour stop one after another keeping the headway 5 meters and form a waiting line. During the first several seconds after the light 
has changed from red to green, the last car of the line should keep standing, and so the cars which are generated and arrive during the same interval should stop and wait. If the last car of the line is moving when new car has arrived, the latter gains the same speed as the former's and occupies the co-ordinates calculated by the equation (1). There are three cases to consider when the light has changed from green to yellow. Those are whether no car exists, or there are some running or still standing on the approach.

Actually, it may not be right to define strictly what behavior a car should assume during yellow hour, but simply we have set up such a model as follows; cars must reform a waiting line no matter whether they are moving or standing if, when the light has changed from green to yellow, they are behind the entrance of the intersection. On the other hand, those which are at or over the intersection at the same time keep running untill they get to the exit co-ordinate. Of course, the cars in the pocket start right turing. This definition may be called in question. Apart from whether or not it is generally lawful, the problems may be of each driver's reaction time and of each car's braking distance. For instance, it is almost impossible for a car, which is running at the speed of $10 \mathrm{~m} / \mathrm{sec}$, to stop at the moment when the driver perceives the light has turned to yellow phase. In this paper, however, we neglect this reaction time in braking, which generally is considerably shorter than that in starting, and further neglect the braking distance of car. Thus every car in the system shall agree to the simple model described above. Fig. 5 shows an example of line of travelling cars just

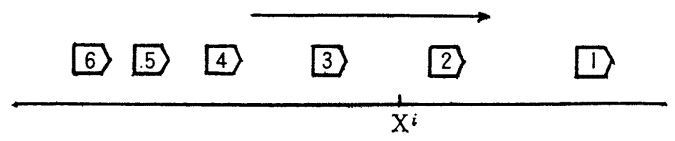

Fig. 5 A line of cars at the end of green light. $X_{i}$ : co-ordinate of the entrance of intersection.

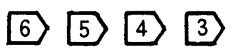

$\mathrm{X}^{i}$

Fig. 6 A reformed waiting line. before the light changes from green to yellow. The line will be reformed into such a waiting line as shown in Fig. 6 at the nextinstant.

Then let's have an explanation about the turning routine. The turning, right and left, cars come to be released out of lane after having run for several seconds. In another words, they are neglected out of the for ward lane the moment when they have reached the middle intersection, and the right turning cars alone come into turning pocket to wait.

By making assumption 3 , we simplified the model as follows, so long as the pocket is not filled with the waiting cars to turn to right, the forward movements of following cars (opposing cars as well) are not to be disturbed at all, otherwise, we imagine those movements are stopped.

\section{TRAFFIC GENERATION}

Before we mention of the block chart for the simulation running, we emphasize the routine for traffic generation.

In general, in order to get traffic, some distribution function of arrivals and certain randum numbers should be given. Well known distribution functions are the normal, binomial, poisson, and exponential ones. What we can get by Monte Carlo Technique with some one of them and the randum numbers is the time interval between arrival. But generally, it is very difficult to determine a satisfactory distribution function of arrivals on actual street, which is equipped intermittently with traffic signals. In such a case, we can use the distribution form, which is acquired by field observation, and some modification of it.

In this simulation test, poor observed date about arrival and speed distributions obliged us to set up such routine for traffic generation as described below.

We can get the probability of an arrival in a unit time from a given hourly traffic volume. Whether there is an arrival in the unit time or not, whether a car arrives at the study area or not, is determined by comparing the probability with a random number distributed in the interval $(0,1)$. The number of arrivals is 0 
or 1 in the unit time, and never more. For instance, if $0<R \leqq p$, there is one arrival, and if $p<R<1$, no arrival, where $p$ means the probability of arrival of a car in the unit time and $R$ is a random number. This routine of traffic generation also includes the routine that distinguishes and memories which a car generated is of right turning, left turning and straight forward cars. In principle, this is the same routine as that mentioned above, i.e., let the turning fraction, right and left, be each $p_{r}$ and $p_{l}$, and the proportion of straight forward cars is given by $1-\left(p_{r}+p_{l}\right)$. So, with the random number $R$ uniformly distributed at $(0,1)$, any one of,

a right turning car if $0<R \leqq p_{r}$,

a left turning car if $p_{r}<R \leqq p_{r}+p_{l}$,

and a straight forward car if $p_{r}+p_{l}<R<1$, is sure to be found. In such a way, we can decide the driving direction of a car.

\section{TESTING PROCEDURE}

Fig. 7 is a block chart showing an outline of testing procedure for a single lane. Here we explain only about this general chart, instead of going into details, which may not be popular. For the 2 lane intersection, the combination of two diagrams, each just like Fig. 7, and for the 4 lane model, the connection of four, each of which is a little different from Fig. 7 owing to several condition added, respectively give us the whole procedures. According to the procedure, we repeat our simulation test once a unit time 1 second.

Now let's have a rough explanation about: Fig. 7.

\section{(1) Initial Setting}

It is necessary for us to set a certain initial state of the model from which we start testing. We have given the model such initial

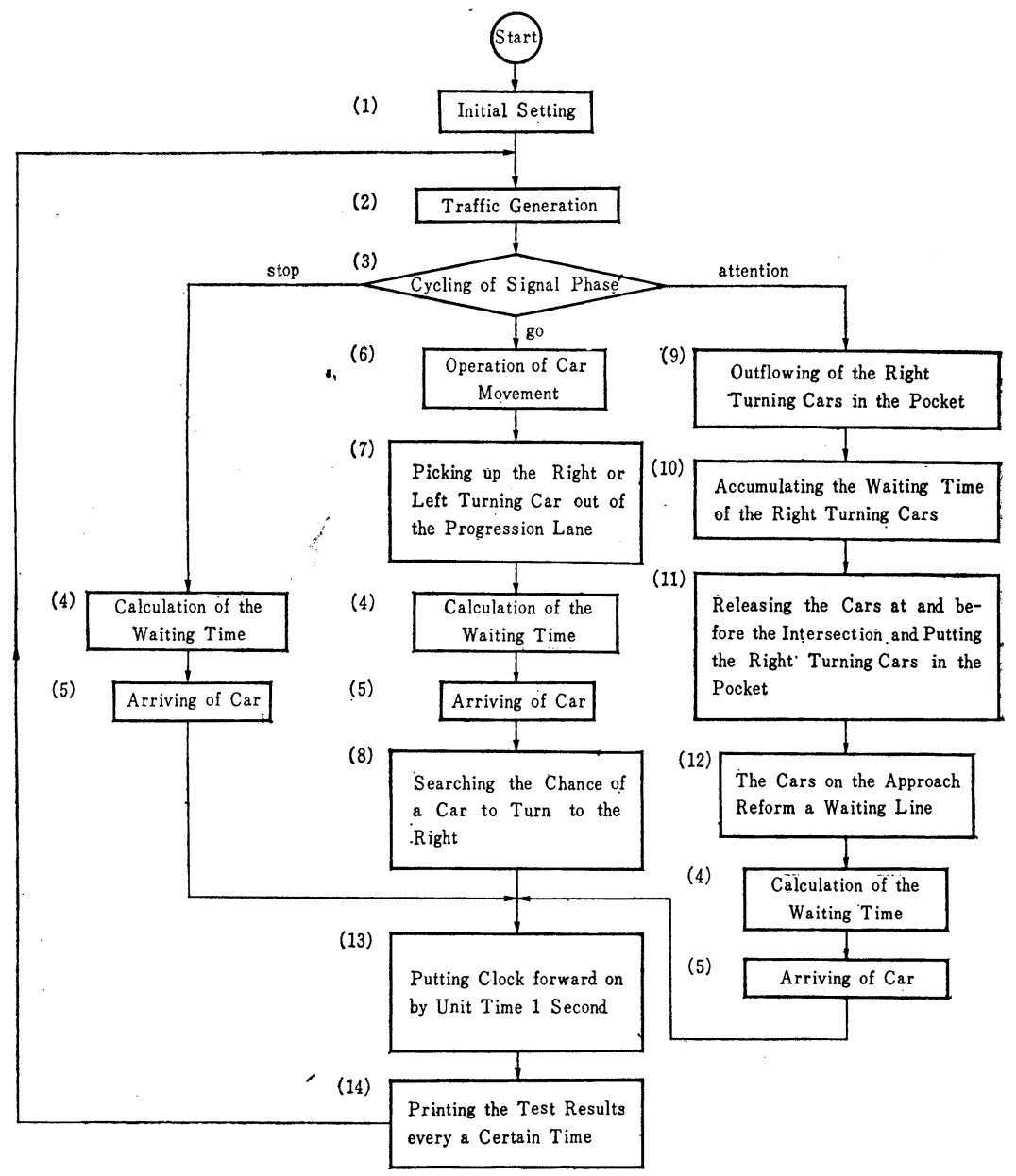

Fig. 7 Block chart (for single lane). 
inputs that there is no car moving or stopped at the intersection and on any approach or lane. Needles to say, we should repeat the test running so many times that the initial inputs should have no influence upon the test results. The data necessary for logical and arithmetical calculation, though not essential to the results, are also to be given as input.

\section{(2) Traffic Generation}

About this we have described in $3 . \quad$ Additionaly speaking, in the 4 lane intersection model, the probability of an arrival in unit time 1 second is given on 2 lanes in a direction.

(3) Cycling of Signal Phase

The signalcycles as follows; red $\rightarrow$ green $\rightarrow$ yellow $\rightarrow$ red $\rightarrow$ green $\rightarrow$ yellow and so on. Table 1 showsseveral kinds of cycle times each of which consists of three phases.

Table 1 Cycle time and phase time.

(in sec)

\begin{tabular}{c|c|c|c}
\hline cycle time phase & stop & go & attention \\
\hline 20 & 10 & 5 & 3 \\
40 & 20 & 15 & 5 \\
60 & 30 & 24 & 6 \\
80 & 40 & 33 & 7 \\
\hline
\end{tabular}

(4) Calculation of the Waiting Time

Here the waiting time means the sum of

i) the time actually stopped by stop phase

ii) the starting delay due to the reaction time

iii) the waiting time of right turning cars in the central pocket.

If there is no intersection, each car is to run on at constant speed of $10 \mathrm{~m} / \mathrm{sec}$, but the intersection with traffic signal forces it such behaviors as

decelerating $\rightarrow$ being stopped $\rightarrow$ accelerating,

or decelerating $\rightarrow$ slowing $\rightarrow$ accelerating.

It therefore is desirable to take account of the whole delay-time resulting from those behaviors. However, suppose we have set up the acceleration or deceleration model, for instance like Fig. 4, and the delay time due to the model will be found to have no relation with the waiting time due to the signal cycle. In another words, the length of waiting line closely contacts with the cycle time, while each car in the line decelerates or accelerates according to the model shown in Fig. 4, and what is essential here is the former. From the standpoint of the optimal cycle time we should pick up only three kinds of waiting times previously mentioned. This, however, never means that we can form the model of acceleration or deceleration at free hand, but that the true waiting time may be obtained by improving those models.

Now, there are several methods of calculating the waiting time. One we have chosen is as follows; the car stopped at a certain time $t$ must stand till the next time $t+1$, and the whole additional waiting time is $N \times 1=N$ seconds where $N$ is the number of cars stopped at time $t$. The total sum of the waiting time occurring in the system is given by the sum of the total before time $t$ and $N$. By this method it is necessary always to remember the total one before time $t$ and the number $N$ of cars waiting in line.

\section{(5) Arriving of Car}

As the approach is only one in a direction in the case of 2 lane model, a car joins to the line of cars according to the rule of arriving mentioned before. In the 4 lane intersection model, there lie two lanes in a direction, and an approaching car should decide which lane to enter. In this case the car joins to the line under the decision rule as follows; if it is the right turning car it runs or stops on the inner lane, if the left turning car on the outer lane, and if the straight going one on the lane on which there exists a shorter waiting line, moreover, it enters the outer when the two lanes have equal length of queue or no car. Of course, in this case as well, the principle described in 2 is to be applied to the speed and the following interval of the new car to keep.

(6) Operation of Car Movement

Our simulation model stands on the basic principle that the drivers should fairly observe the signal sign. So it is natural to assume that the car at the head of the waiting line is sure not to start before 1 second, reaction time, has passed after the signal has changed from red to green. 
Let's explain the concrete way of giving forward movements of cars on lane. Let's remark a certain car on lane, the new co-ordinate of the car is given by

$$
x(t+1)=x(t)+\dot{x}(t)
$$

where $x(t)$ is its co-ordinate at time $t, x(t+1)$ is the new co-ordinate to reach at time $t+1$, and $\dot{x}(t)$ is its speed at time $t$ (accurately the average between $t$ and $t+1$ ), i.e., when the car, from equation (1), finds forward movement $\dot{x}(t)$ possible, it determine to move by distance $\dot{x}(t) \times 1$ from the present co-ordinate $x(t)$ to the new position $x(t+1)$. The modification of the contents in the computer memory is performed in such a way as mentioned already.

(7) Picking up the Right or Left Turning Car out of the Progression Lane

We give up further details, because we have, though may rather roughly, mentioned before.

(8) Searching the Chance of a Car to Turn to the Right

The car looking forward right turning in the pocket near the middle of the intersection can finish its turning, when it finds out 5 second or more headway on the opposite lane. That headway, of course, should be found to be across the imaginary turning lane across the counter-lane. If there is no car on the counterlane behind the intersection, whether the car under study is to start or not depends on the traffic generated through a temporarily set routine. In any case, the chance of right turning is only in the hands of the straight forward cars on the opposite lane. What is just mentioned takes effect during just green hour.

(9) Outflowing of the Right Turning Cars in the Pocket

This is the routine through which cars in the pocket start and finish right turning one after another during attention phase. This routine as well as the others is passed through once a unit time 1 second.

(10) Accumulating the Waiting Time of Right Turning Cars

This routine includes almost the same operation as described in (4) of this section except the point that this is given to the cars standing in the pocket during yellow light.
(11) Releasing Cars at and before the Intersection and Putting the Right Turning Cars in the Pocket out of the System.

This routine comes into effect only for the first 1 second after the light has changed from green to yellow. Indeed, not only for the first 1 second but for the first several seconds, those cars running at and before the intersection will continue to run till they come to be released out of the lane or lanes. However, so long as this simulation model is concerned, it is of no essential importance to follow after these real behaviors. From standpoint of machine time required, it is obvious that this model in which those cars are released out of the lanes at the first scanning is of much advantage, because by clearing out all of those cars at once the after operation can be saved. In that clearing operation, the right turing cars, if any, should be picked up and put into the pocket. If there has been no car in the pocket, the first car which happened to be let in it can turn right away so that there should be no waiting time added. Otherwise, however, the car must wait until those cars which has been standing there have finished turning.

(12) The Cars on the Approach Reform a Waiting Line.

We already explained about the outline by Fig. 5 and Fig. 6. Here, we want to have a little more explanation. In Fig. 5, there are four cars running figured by $1,2,3$, and 4 , and car 5 is supposed to be starting at next time. Finding the yellow light, cars $3,4,5$, and 6 which have been still on the approach behind the intersection should stop and wait. Then a waiting line will be reformed behind $X^{i}$, the co-ordinate of the entry of the intersection. This operation is to be performed at the first time when the signal changes to attention phase. And the other succeeding operation can be saved. Fig. 6 shows a reformed queue in which cars 1 and 2 seen in Fig. 5 don't exist because they have been cleared out.

(13) Putting Clock forward on by Unit Time 1 second.

As mentioned before, we have set 1 second as the unit time every which the whole system 


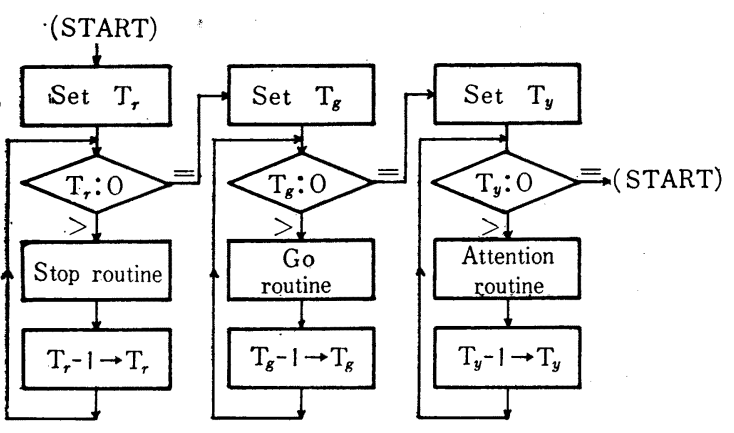

Fig. 8 . Flow chart for signal operation.

is scanned. In another words, the operations of whole system including traffic generation, operation of forward movements of cars, calculation of the waiting time, and so on are repeated every 1 second. The clock operation, therefore, is of no essential part. What we mean by putting clock forward is the operation of signal change. Now let's refer to Fig. 8 about this signal operation.

In Fig. $8, T_{r}, T_{g}$, and $T_{y}$ show respectively the stop, go, and attention phase times (in second), and $T_{r}+T_{g}+T_{y}$ gives one cycle time. Those which are named the stop, go, and attention routines in Fig. 8 mean respectively those which branch from signal box (3) in Fig. 7. At the choice box $T: 0$ the computer finds the routine to pass through, what operation to execute. For instance, when it finds $T_{r}>0$, it determines to go through the stop routine since $T_{r}>0$ means the light keeps red. Everytime when it passes through the stop routine, the computer memories $T_{r}-1$ as new $T_{r}$ in the same memory register as before and goes back to the choice box above. The computer goes round the same routine until it finds $T_{r}$ is equal to zero, stop phase is over. Then it jumps to new routine, i.e., the go routine, in which in the first place it gives a certain phase time to $T_{g}$ and next goes round the go routine. Of course, every time it stops at the choice box it examines if $T_{g}$ is positive and memories $T_{g}-1$ as new $T_{g}$. By way of attention routine, the computer goes back to the stop routine to repeat the same circulation.

(14) Printing the Test Results every a Certain Time.

The test results are to be printed three times a signal cycle, at the end of each phase. The printed results include, on each lane,

i) the total number of cars generated (in vehicle)

ii) the total number of cars stopped (in vehicle)

iii) the queue length at the time (in vehicle)

iv) the total waiting time (in second)

v) the number of cars waiting to turn to right (in vehicle)

vi) the total waiting time of the right turning cars stopped in the pocket (in second)

vii) the total numper of the right and left turning cars generated

viii) Average waiting time as the function of the total number of cars generated (in sec/veh).

\section{TEST RESULTS AND THEIR ANALYSIS}

The results obtained by this test are put together into Fig. 9. Each curve has been drawn by adapting a suitably smooth curve to the average waiting times dotted for each cycle time. It is the fact that the hourly traffic volume in the 4 lane model has been given on 2 lanes in each direction. In Fig. 9, however, it is converted to the one on a single lane. From Fig. 9 may follow those as below.

traffic volume (vehicles/hour/lane)

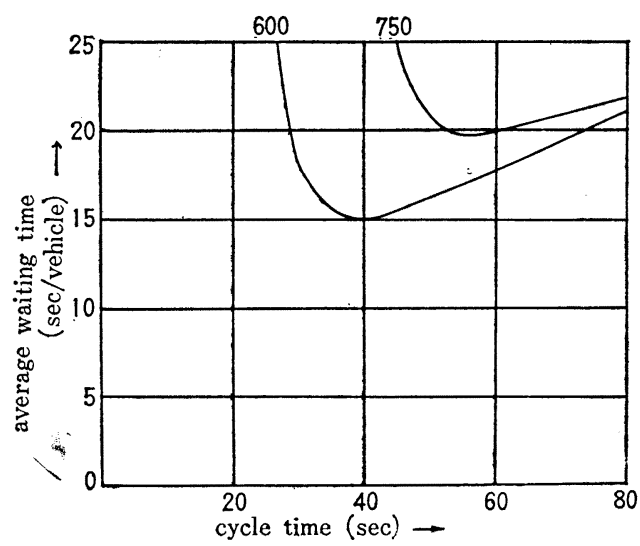

Fig. 9 Average waiting time as a function of cycle time and traffic volume. (turning fractions, right and left, each 15\%). 
1) At the intersection with hourly traffic volume per lane of about 600 vehicles, nearly 40 seconds gives the optimal cycle time to minimize the average waiting time. The practical cycle time may be about 50 seconds.

2) Similarly, in the case of about 750 vehicles, the optimal cycle time should be given by nearly 55 seconds and the practical one by about 60 or a little more seconds.

3) The hourly traffic volume per lane of 1,000 vehicles or more will require the cycle time longer than 80 seconds.

4) The cycle time shorter than 30 seconds should be impotent even where the traffic volume is of about 600 vehicles per hour per lane. And such a traffic light may be worse than nothing for it perhaps fails in better controlling of traffic flow.

The other conclusions have been reached as follows.

5) The lighter the traffic volume is, the shorter the cycle time can be. The cycle time shorter than 30 seconds, however, had better be not used.

6) As the cycle time is shorter, the starting delay of the leading car causes the larger traffic delay, to which we should pay attention especially when the traffic consists of various kinds of vehicles.

7) From the practical point of view, the cycle time should be a little longer than the optimal obtained by test. It is true that the waiting time increases with the slow gradient for the cycle time longer than the optimal, while it makes remarkably rapid increase for that below the optimum.

8) The shorter the cycle time is, the less disturbance the right turning car gives to the movements of the following cars. In this simulation the turning fractions, left and right, have been set always constant each with $15 \%$ of whole traffic in a direction. The number of the right turning cars standing in the pocket has been found always under the capacity when the cycle time is shorter. That is obviously because only small number of the right turning cars is allowed to flow into the intersection during the shorter green hour. Alternatively, for the cycle time over 60 seconds the capacity has sometimes or rather frequently been found too little.

9) As the cycle time is longer, the greater congestion will be caused by the right turning cars waiting in the pocket. Therefore, at the intersection through which passes heavy traffic volume with the larger turning fraction, especially the right turning one, we should take some kinds of traffic regulations, for instance, control of right turning.

10) The complete prohibition of right turning may increase the total traffic in whole networks, so it seems desirable to cut and divide the turning fraction into the smaller ones. This means, for instance, that we regulate so that at a certain intersection a given kind or kinds of cars may finish their right turning and at another one other kinds may do.

11) So far as this simulation results are concerned, the practical capacity (in vehicles per hour per lane) of at-grade intersection equipped with traffic light is estimated to be about 800 .

12) The machine time by the physical representation was about 4 times as much as that by the memorandum representation.

\section{CONCLUSIONS}

We have mentioned of the outline and some results of our traffic simulation, which seems to include several questions to solve and improve. We give some comments about them.

1) To simulate the orthogonal traffic. Our test results have an effect only at those intersections through which equal traffic volume passes each in a direction and in the orthogonal, otherwise have not.

2) To have usefull data about the influence of the right and the left turning cars on the forward movements of the following cars.

3) To formulate de- and accelerating and slowing behaviors. By this we may find the more accurate delay time or waiting time.

4) Ultimately, to carry out the simulation of traffic flow on the whole networks. We should remember the improvement of traffic condition at a single intersection does not always 
optimize the whole system.

5) The right turning routine, reaction time and so on are still to be discussed about from human engineering and psycological points of view.

6) To determine and improve the distribution function of arrivals by observation and theory. On a practical street, completely randum arrivals are hardly to be expected.

\section{References}

1) T. Sasaki and T. Fujii : "Traffic Simulation at a Weaving Section", The Summary of Lectures in the 15th Annual Meeting of the Japan Society of
Civil Engineers, pp. 49-50, May, 1960.

2) S. Watanabe and T. Fujii : "On Traffic Simulation at an At-grade Intersection", The Summary of Lectures in the 16th Annual Meeting of the Japan Society of Civil Engineers, pp. 33-36, May, 1961.

3) A. Kato and S. Myozin : "Traffic Simulation at a Signalized Intersection", The Summary of Lectures in the Annual Meeting of the Kansai District Branch of the Japan Society of Civil Engineers, pp. 141-142, November, 1962.

4) E. Kometani and S. Myozin : "Traffic Simulation at a Signalized Intersection $\cdots 4$ lanes...", The Summary of Lectures in the 18th Annual Meeting of the Japan Society of Civil Engineers, pp. 93-94, May, 1963.

(Received Feb. 9, 1965) 